
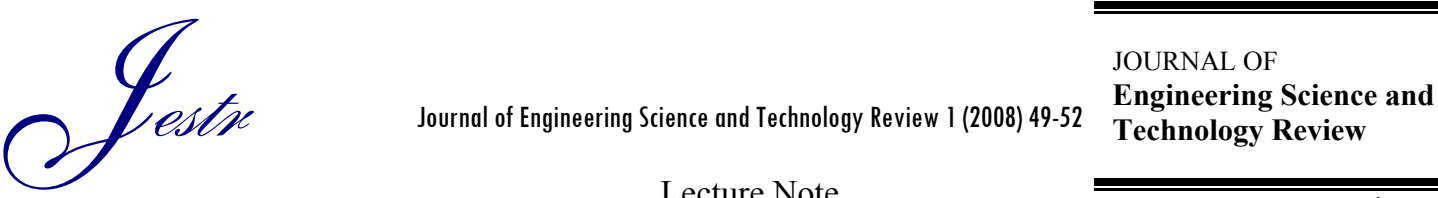

Lecture Note

www.jestr.org

\title{
The human growth and the healthy environment as a condition of balanced de- velopment for the course of human.
}

\author{
I. Koukoumpliakos*,1 ${ }^{*}$ and M. Nousia ${ }^{2}$ \\ ${ }^{1}$ Greek Open University, Department of European Culture, Nikiforou Foka 37str. 412.23 Larissa. \\ ${ }^{2}$ Agriculturist, MSc, Kranonos 3 str,.412.22 Larissa, \\ Received 14 April 2008; Accepted 17 September 2008
}

\begin{abstract}
The rising standard of living has evoked an important burden in the natural environment leading to an immense deterioration of nature. The pollution of the atmosphere and seas, the industrial waste, the climatic changes, the chaotic built-up extension threaten the health of all of us. The responsibility for the pollution of the environment is allocated in everybody. The State is accountable not only for the political planning and the policy that practises, but also for the frame it shapes for the citizens to follow. The improvement of the quality of environment must be combined with the economic progress and growth. The intensity of environmental problems worries the International Community, while the saving and growth of new green forms of energy appear as imperative need. We find already ourselves in a progressive but continuous growth, which has the conditions to develop in the future with spectacular rhythm.

The methodology of present work is recommended for: a) bibliographic research, mainly Greek, b) comparison of the facts that are exported from research, connecting them with the given facts. This comparison leads to the necessary coexistence of a healthy environment and a viable growth.

In the results of the research the essential conditions of coexistence are presented between these two. Thus we realise that: a) the configuration of an evener environmental conscience is required via the education which would guarantee the balanced and sustainable growth, b) the change of perception of people as lords of nature is considered necessary and its replacement by the awareness that the nature does not constitute an inexhaustible resource.

With that in mind we conclude that: a) the research is required to lead to alternative sources of energy such as the creation of Aeolian parks. It is estimated that more investments in other types of renewable sources of energy such as geothermal and hydroelectric can constitute also advisable solutions. The use of renewable sources of energy limits the use of mining fuels, allows the reduction of emissions, leading to a smaller dependence on imported fuels and other pollutants b) the applications of policies such as the biological cleaning and the recycling of materials like paper, glass, plastic and aluminium are necessary, c) the protection of nature which will aim at the supervision of biodiversity for the survival of species that are in danger in order to maintain the balance of flora and the fauna of planet is considered mandatory and d) finally there is necessity of an evener activation of management and protection institutions of the environment with staffing and training of control services.
\end{abstract}

Keywords: Viable growth, energy, environment, Aeolian parks.

\section{Introduction}

The requirements of modern life and the increasing predominance of the human being on nature led to the excessive bloodletting of our natural wealth, so that the balance of flora and fauna of the environment is threatened. The pollution of the atmosphere and seas, the industrial waste, the climatic changes, the anarchic built-up extension threaten the health of all of us. The improvement of quality of environment is required, combining itself with the economic progress and growth, and more specifically the viable growth. Viable growth constitutes the effort to satisfy

\footnotetext{
* E-mail address: jiovanil@hotmail.com

ISSN: 1791-2377 (C) 2008 Kavala Institute of Technology. All rights reserved.
}

the needs of the present generations, without mortgaging the possibility to satisfy the needs of the future ones. (Brutland, B. H., [1]).

\section{Methodology}

The methodology that is followed in this particular assignment is supported by bibliographic research that connects with the facts that are exported from the study. This connection leads to the cross-correlation between the growth of the human factor and the natural environment.

The viable growth includes two terms: the term growth and the term viable. Growth is a self-supplying process that develops with the differentiation of economic activities, increases the wealth, giving thus the possibility in a big number of individuals to improve their living conditions. In 
order for growth to be viable, it needs to be articulated around the term viability (Brunel S., [2]). Viability is distinguished in absolute and relative. Absolute viability gives priority to the environment, while relative viability deals with the fact that we can substitute the natural capital with artificial (Mancebo F., [3]).

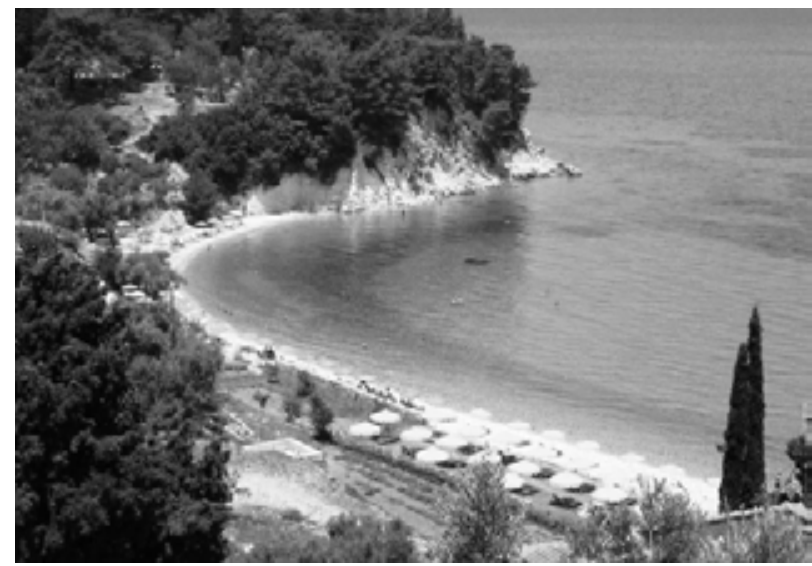

Fig 1. Balanced Growth

In the last years the environment constitutes a point of reference for the industrial and technical culture (Gkorz P., [4]). Society felt the need to exploit the resources of natural environment without restrictions destroying and polluting it (Gikas P.,[5]). However, the 1990s were characterized by an ecological intervention (Rossi G., [6]). According to the Brutland report [1] two basic ideas of ecology entered dynamically in the international scene: a) Since the protection of the planet constitutes our biggest priority; we ought to adopt ways of production that would respect more the environment, b) the western way of life and consumption cannot extend itself in the rest of the world, without seriously threatening the future of the planet (Brunel S., [2])

\section{Results - Discussion}

The forest exploitation involved progressive deforestation which resulted in a decrease in the resistances of nature toward unfavourable meteorological phenomena. Thus, the floods, whose waters used to be absorbed by the forest, are now retained with difficulty. The uncontrolled floods led also to other phenomena such as the rise of the level of the sea, which also created other specific problems. It has been observed that due to the rise of the level and the speed of water, small fish as well as their eggs are being carried away. On the other hand the reduction of the water level, which has been observed in periods of intense drought, has made it difficult for the fish to find spaces for reproduction. Therefore the maintenance of expected level of water constitutes an important condition for the balance of marine environment (Neofitou X., [7]).

The water resources, that constitute an important element of economic growth, safeguard the ecological balance. However, a lot of marine ecosystems have been differentiated from their natural state due to the permanent pollution of the seas (see fig 2) which has resulted in several species of flora and fauna to be threatened nowadays with disappearance. Therefore complete protection of marine ecosystems is essential for the preservation of the biodiversity, the avoidance of floods and the control of the pollution of the seas (Neofitou X., [7]).

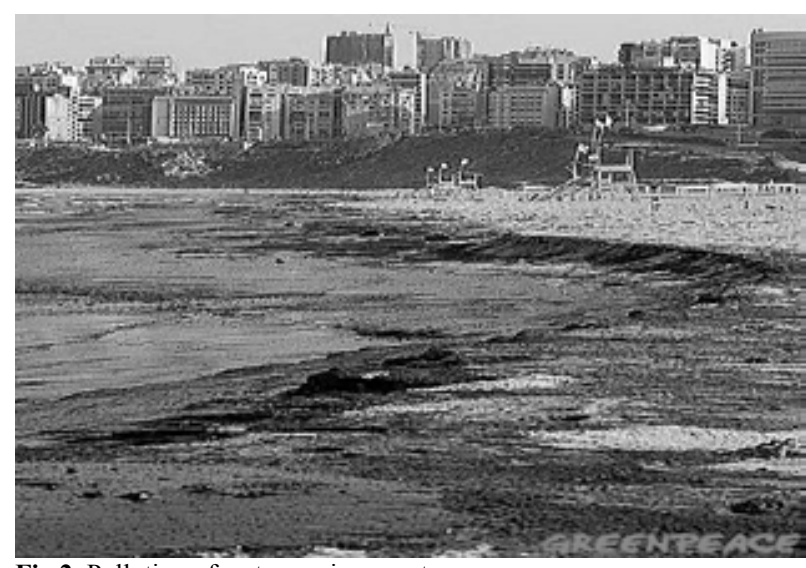

Fig 2. Pollution of water environment

We all recognize the damaging contribution of car in the emission of poisonous pollutants. The carbon dioxide that is emitted aggravates considerably the atmosphere. We can contribute all in this by limiting our unnecessary moving. In our commuting to work we can use the mass transports. All of us will not only have to turn ourselves into the new hybrid type of cars (see fig 3), which are estimated to be friendly to the environment, but also to the acquirement of a circulatory culture

(http://www.studentguru.gr/blogs/teohaikalis/archive/2007/0 2/04/555.aspx [8]).

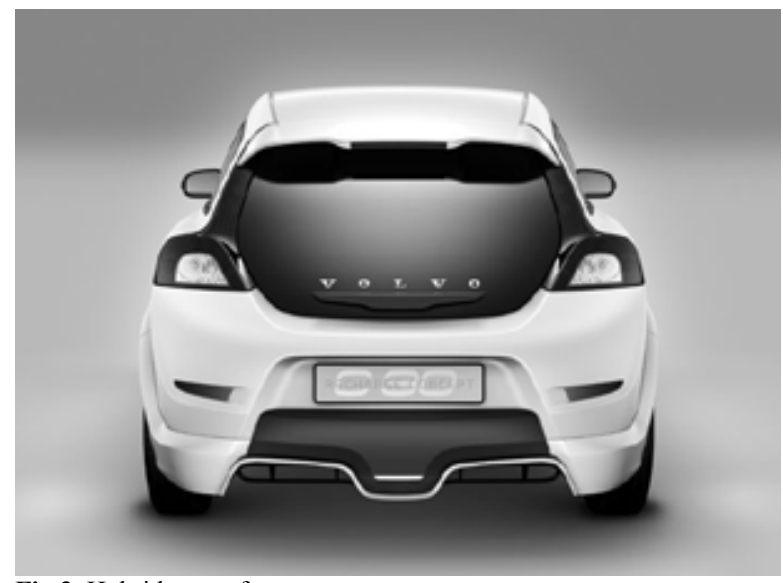

Fig 3. Hybrid type of car

The bicycle is another advisable solution for transportation in the cities. The use of bicycle helps the environment and our everyday routine, while at the same time it upgrades the quality of life in the cities. Naturally in order for all of these to happen, a change of mentality is required. The right environmental education for example, could be turned into an advantage in the future. A higher absorption rate of professors of environmental education by the school system is required, so that the students obtain the right education for the subject. At the same time social and political will are required, as well as the right attitude which could overcome any stagnancy for the matter.

It constitutes an obligation for all of us to focus on issues of recycling materials, separating the litter per category of products, initially in domestic level and then in every enterprise. In this way paper, glass, aluminium and plastic can be recycled. Also we owe to turn into the production of products of biological culture that have been already proved friendly to the environment. 
It is advisable that programs aiming at the creation of such technology be financed that would allow the recycling and exploitation of the heat losses of factories. One potential solution is the placement of turbines in the chimneys of the factories, which will produce energy from the exhaust fumes (see fig 4). In this way we could also limit the emissions of greenhouse gases.

(http://www.tovimadaily.gr/SectionList.aspx?d=20071116\& nid=6599541\&sn=\&spid=916 [9]).

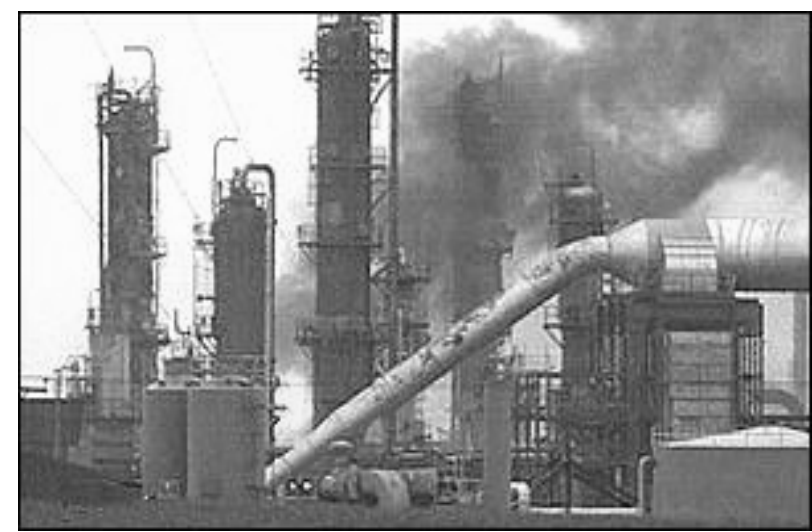

Fig 4. Exhaust fumes from factories

At the same time the scientific research owes to be led to alternative sources of energy like for example the creation of Aeolian parks (see fig 5). The use of Aeolian energy constitutes a friendly to the environment energy choice. The wind offers an inexhaustible source of energy, which is, of course, provided free of charge. It protects the Earth as each kilowatt-hour that is produced by the wind replaces one that is produced by conventional stations which pollute the atmosphere with greenhouse gases. In other words wind energy does not overload the local environment with dangerous gasses, carbon monoxide, sulphur dioxide, cancer-causing molecules etc., as is the case with the conventional stations of electric energy production, while finally it helps in the decentralisation of the energy system decreasing the amount energy that is lost in the distribution. (http://www.cres.gr/kape/publications/papers/dimosieyseis/p aper\%2019.doc[10]).

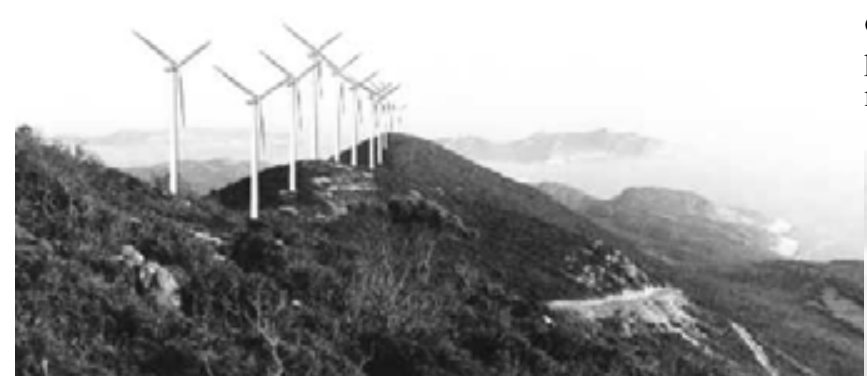

Fig 5. Aeolian park

$80 \%$ of the quantity of energy that is used in our days emanates from deposits of mining fuels or from uranium. Mining fuels include the coal, the oil and the natural gas. These deposits are already found in limited quantities. On the contrary energies that emanate from the sun, the wind, the waterfalls, and the earth's heat are renewable sources. These forms of energy have as their basic sources, the sun and the earth. Many characterize as renewable the energy that springs from the waste considering that the duration of human or animal activity is continuous. Consequently it constitutes a source of non-stop renewable waste. According to many the term biomass includes the energy coming not only from plants but also from any waste of human activity. (Vernier I., [11]).

Recent studies show that oil reserves suffice for approximately another 40 years based on the current consumption, natural gas for about 65 years, while the coal roughly for about 200 years. This means that alternative solutions are required. The means of mass transport for example could stop consuming oil exclusively. A suitable solution would be the consumption of a new fuel, methanol, which is produced by coal. The combustion of mining fuels leads to the overheating of the Earth (the greenhouse effect) because of the carbon gasses that are emitted by their combustion in the atmosphere (Vernier I., [11]).

It is advisable that new policies are applied which will protect the countryside and the regional growth. The reinforcement of the business dexterity and competitiveness should be formed with the attendance of the regional authorities (Zamanidis P.,[12]). In this way the rural population remains in its region, the employment is strengthened, the comparative advantages of each region appear and the environmental infrastructure of the prefecture is improved. However specific credits are also required. Any ameliorative interventions for the countryside should aim at the logic of sustainable growth. (Papavasileiou G., [13]).

\section{Conclusion}

It is essential that the public be informed on the multiple benefits of the renewable sources of energy so that the local protests against investments on wind generators and hydroelectric power stations are not observed anymore. The State should also include in the budget expenses for subsidies for investment plans that have been submitted for exploitation of renewable sources of energy. The responsibility for the pollution of environment is allocated in all. New innovations are required that can be spread fast and easily without any distance and border restrictions (Jessua C., [14]). The State is accountable for its political planning and the policy which practises as well as for the frame which is shaped by it and which the citizens should follow. Besides, according to Giousernar M., [15] "humanity is not only threatened with disappearance on a dead planet. Every person needs essential air, suitable soil, education and meaning in life in order to live humanly."

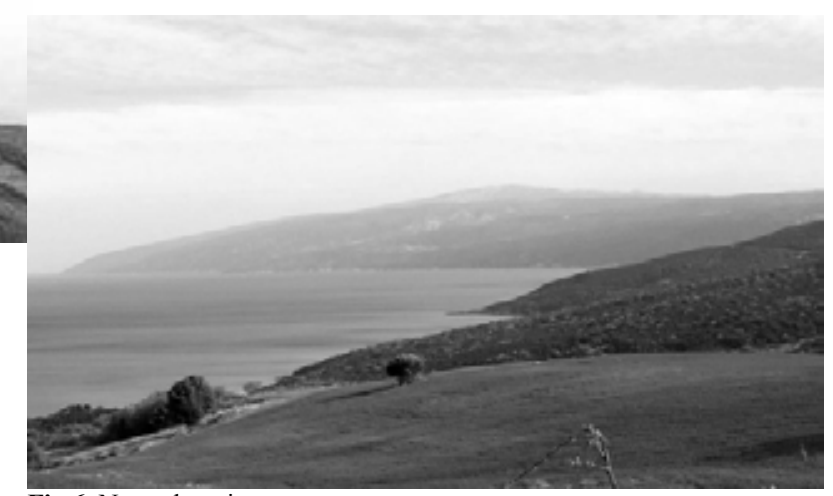

Fig 6. Natural environment 


\section{References}

1. Brutland Report, Sustainable Development, (1987)

2. Brunel, S., The viable growth. Publications Gnosi. Athens, (2007)

3. Mancebo, F., Le development durable. A. Colin, (2006)

4. Gorz, P., L' environment. Publications Gnosi. Athens, (1971)

5. Gkikas, P., Lexiko Ennion Genikis Paideias. Publications Savvalas. Athens, (1995)

6. Rossi, G., L ingerence ecologique CNRS (2000)

7. Neofitou, Hr., Biological Protection and Management of Marine Ecosystems, Geotechnical Briefing, 149: 25-28 (2007)

8. http://www.studentguru.gr/blogs/teohaikalis/archive/2007/02/04/5 55.aspx

9. http://www.tovimadaily.gr/SectionList.aspx?d=20071116\&nid=65 99541\&sn=\&spid=916
10. http://www.cres.gr/kape/publications/papers/dimosieyseis/paper\% 2019.doc

11. Vernier, I., The renewable sources of energy. Publications Gnosi. Athens (2007)

12. Zamanidis, P., Problems-Perspectives of growth in the primary sector in Western Macedonia, Geotechnical Briefing, 149: 10 (2007)

13. Papavasileiou, G., Institutional and professional exploitation of geotechnical in the frames of viable growth of cities and countryside, Geotechnical Briefing, 149:7-9 (2007)

14. Jessua, C., Capitalism. Publications Gnosi. Athens (2007).

15. Giousernar, M., I charistiki voli. Publications Hatzinikoli. (1980) 